\title{
Stress during puberty boosts metabolic activation associated with fear-extinction learning in hippocampus, basal amygdala and cingulate cortex
}

\author{
Maria Toledo-Rodriguez ${ }^{\mathrm{a}, *}$, Alain Pitiot ${ }^{\mathrm{b}}$, Tomáš Paus ${ }^{\mathrm{b}, \mathrm{c}}$, Carmen Sandi ${ }^{\mathrm{d}}$

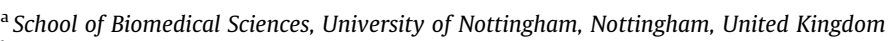 \\ ${ }^{\mathrm{b}}$ School of Psychology, University of Nottingham, United Kingdom \\ ${ }^{\mathrm{c}}$ Rotman Research Institute, University of Toronto, Toronto, Ontario, Canada \\ ${ }^{\mathrm{d}}$ Laboratory of Behavioral Genetics, Brain Mind Institute, Ecole Polytechnique Federale de Lausanne (EPFL), Lausanne, Switzerland
}

\section{A R T I C L E I N F O}

\section{Article history:}

Received 15 February 2012

Revised 20 May 2012

Accepted 28 May 2012

Available online 1 June 2012

\section{Keywords:}

2-Deoxyglucose

Adolescence

Brain metabolism

Fear memories

Gender differences

Stress

\begin{abstract}
A B S T R A C T
Adolescence is characterized by major developmental changes that may render the individual vulnerable to stress and the development of psychopathologies in a sex-specific manner. Earlier we reported lower anxiety-like behavior and higher risk-taking and novelty seeking in rats previously exposed to peripubertal stress. Here we studied whether peri-pubertal stress affected the acquisition and extinction of fear memories and/or the associated functional engagement of various brain regions, as assessed with 2-deoxyglucose. We showed that while peri-pubertal stress reduced freezing during the acquisition of fear memories (training) in both sexes, it had a sex-specific effect on extinction of these memories. Moreover hippocampus, basal amygdala and cingulate and motor cortices showed higher metabolic rates during extinction in rats exposed to peri-pubertal stress. Interestingly, activation of the infralimbic cortex was negatively correlated with freezing during extinction only in control males, while only males stressed during puberty showed a significant correlation between behavior during extinction and metabolic activation of hippocampus, amygdala and paraventricular nucleus. No correlations between brain activation and behavior during extinction were observed in females (control or stress). These results indicate that exposure to peri-pubertal stress affects behavior and brain metabolism when the individual is exposed to an additional stressful challenge. Some of these effects are sex-specific.
\end{abstract}

(c) 2012 Elsevier Inc. All rights reserved.

\section{Introduction}

Adolescence is a critical developmental period characterized by physical, hormonal and behavioral changes. During this transition, region-specific changes in brain structure and function take place (Paus, Keshavan, \& Giedd, 2008). One of the major landmarks of adolescence is the attainment of sexual maturity (gonadarche) that is paralleled by the emergence of sex differences (Styne, 1994). These sex differences do not only involve sexual and reproductive functions, but also behavioral and hormonal responses to stressful circumstances (Dahl, 2004). Indeed in humans, adolescence is also accompanied by sharp and sex-related raise in morbidity and susceptibility to psychopathologies (Kessler, 2003; Kessler \& Magee, 1993; Penza, Heim, \& Nemeroff, 2003; Steinberg et al., 2005). Furthermore, stressful experiences during childhood and adolescence increase vulnerability for developing psychiatric disorders later in life (Heim, Plotsky, \& Nemeroff, 2004; Kessler \& Magee, 1993; Penza et al., 2003). Moreover, heightened task-related brain

\footnotetext{
* Corresponding author. Address: School of Biomedical Sciences, Medical School, Queen's Medical Centre, Nottingham NG7 2UH, UK.

E-mail address: maria.toledo@nottingham.ac.uk (M. Toledo-Rodriguez).
}

activity during adolescence appears to predict the development of psychopathologies later in life (Sabb et al., 2010).

In the rat, adolescence is considered to last from postnatal days 21-28 till postnatal days 42-59 (depending on the authors; (Spear, 2000; Spear \& Brake, 1983; Tirelli, Laviola, \& Adriani, 2003)). A number of animal models have been developed to study the short and long-term impact of stress during adolescence (Isgor, Kabbaj, Akil, \& Watson, 2004; Ito, Nagano, Suzuki, \& Murakoshi, 2010; McCormick, Smith, \& Mathews, 2008; Sterlemann et al., 2010; Toledo-Rodriguez \& Sandi, 2011) and during the juvenile period (Avital \& Richter-Levin, 2005; Jacobson-Pick \& Richter-Levin, 2010). Recently, we have shown that stress right before and during puberty lowers anxiety and augments novelty-seeking behavior but does not affect 'depressive-like' behaviors in adolescent rats (Toledo-Rodriguez \& Sandi, 2011). This reduction in anxiety could be due to long-term changes in activation of the limbic system and might affect future emotional learning. Pavlovian fear conditioning is a well-validated test of emotional learning and memory (Maren, 2001). During conditioning the individual develops a conditioned response (CR; e.g. lack of movement or "freezing" in the rodent) when a non-threatening conditioned stimulus (CS; e.g. sound for auditory fear conditioning) is paired with an aversive 
unconditioned stimulus (US; e.g. mild electric foot shock in many rodent studies). If after conditioning the CS (sound) is presented multiple times in the absence of the US (foot shock), the CR (freezing) eventually diminishes or extinguishes (phenomenon known as extinction). Fear extinction (also known as conditioned emotional response) is an active learning process that allows adaptive control of conditioned responses.

The neuronal circuitry of fear conditioning and extinction includes hippocampus, prefrontal cortex and amygdala (Maren, 2001; Rauch, Shin, \& Phelps, 2006). The use of ${ }^{14} \mathrm{C}$-2-deoxyglucose (2DG), a radiolabeled glucose analog, enables autoradiographic visualization of regions metabolically, in relation to brain activity, in freely moving animals (Sokoloff, 1992). This is possible because, during glycolysis, 2DG is trapped within active brain cells when it is phosphorylated by hexokinase (Sokoloff et al., 1977). So far, the majority of studies imaging metabolic activation during extinction in rodents have focused on males (mainly adults) (Bruchey, Shumake, \& Gonzalez-Lima, 2007; Hefner et al., 2008; Herry \& Mons, 2004; Muigg et al., 2008). Yet it is becoming increasingly clear that females differ from males in their morphological and behavioral responses to stress and brain response to various stimuli (Garrett \& Wellman, 2009; Maeng, Waddell, \& Shors, 2010).

Here we investigated, during late adolescence, whether peripubertal stress affected in a sex-specific manner: (a) the acquisition and extinction training of fear memories and/or (b) regional brain metabolism during extinction training. For this purpose, we examined the differences in 2DG uptake associated with an exposure to peri-pubertal stress between "quiet" control animals and animals undergoing extinction of fear memories. Both males and females were studied and we carried out parallel assessments of metabolic activity in the brain regions previously shown to be involved in emotional control and memory: frontal cortex (including cingulate, insular, orbital, prelimbic, infralimbic and motor cortices); hippocampus (including entire hippocampus, CA1, CA3 and dentate gyrus); amygdala (including basolateral, basal, lateral and central amygdala) and the paraventricular nucleus of the hypothalamus. Finally, we correlated the metabolic activation in the different brain structures with the behavior of the animal during extinction.

\section{Materials and methods}

\subsection{Animals}

Subjects were the offspring of rats purchased from (Charles River Laboratories, France): 37 male and 35 female Wistar Han rats weaned at postnatal day 21 . Rats from different litters were mixed, placing equivalent numbers of animals from each litter in the stress and control groups. Rats were housed in same-sex standard plastic cages ( 3 or 4 per cage) on a $12 \mathrm{~h}$ light-dark cycle (light on at 7:00 AM). Food and water were available ad libitum. All the procedures described were conducted in conformity with the Swiss National Institutional Guidelines on Animal Experimentation, and approved by the Swiss Cantonal Veterinary Office Committee for Animal Experimentation. In a few cases, some of the brain tissue was damaged due to freezing artifacts, folds, tears or air bubbles. This led to up to three rats (maximum two per group) to be excluded from the analysis of a small number of brain regions.

\subsection{Peri-pubertal stress procedure}

The peri-pubertal stress protocol used was described previously (Toledo-Rodriguez \& Sandi, 2011). In brief, rats (18 males and 17 females) underwent stress during postnatal days 28-30 (three consecutive days pre-puberty), 34, 36 (the latter 2 days corresponding to the period of female puberty), 40 and 42 (corresponding to the period of male puberty). The stressors were: (a) placement for 5 min in a novel environment (only the first day of stress), (b) exposure to trimethylthiazoline (TMT) odor (Phero Tech Inc. Delta, Canada) during $25 \mathrm{~min}$, (c) placement on an elevated platform during 25 min under direct bright light. After stress, rats were placed for $15 \mathrm{~min}$ in a clean cage without sawdust fitted with perforated Perspex partitions. In this way, animals could see, hear and smell, but not touch, each other. The timing of the stress was random (unpredictable to the animal) but always administered during the light period. During the days of stress, control rats (18 males and 17 females) were manipulated for 2 min by the experimenter.

\subsection{Experiments}

Two independent experiments were performed. Experiment 1 investigated whether stress during adolescence affected brain metabolism during a recall of a stressful memory. In this case, rats underwent Auditory Fear conditioning (AFC) training and test before mapping brain metabolism (AFC, $n=8$ control male, nine control females, 10 stressed males and eight stressed females). Experiment 2 studied whether the effect of stress during adolescence on brain metabolism could be generalized to non-stressful situations. In this case, rats were left undisturbed in their home cages (basal, $n=10$ control males, eight control females, eight stressed males and nine stressed females).

\subsection{Fear conditioning}

Training and testing took place in three identical rodent observation cages $(30 \times 37 \times 25 \mathrm{~cm})$ placed into a sound-attenuating chamber, illuminated with a $20 \mathrm{~W}$ bulb. Ventilation fans provided background noise of $68 \mathrm{~dB}$ (the whole Fear System was acquired from Panlab, Spain). Rats were transported from the colony room to the adjacent fear conditioning room where training and testing took place. After each testing session, animals were returned to their home cages. The rats' behavior was recorded and later scored with in-house produced behavior observation software (Clicker v1.13) by an observer blind to the treatment or sex. Freezing behavior served as a measure of conditioned fear. Freezing was defined as behavioral immobility except for respiration movements for at least $2 \mathrm{~s}$. Freezing times were automatically transformed to percentage freezing values. Below there is a detailed description of the experimental schedule used:

\subsubsection{AFC training}

Four days after the termination of the stress procedure stressed and control rats (46 days old, experiment 1) underwent AFC training. Rats were exposed to Context A (black walls of smooth texture, steel grid floor, cleaned with $2 \%$ ethanol) during $160 \mathrm{~s}$, followed by three presentations of tone-shock pairings where the tone (lasting $20 \mathrm{~s})$ co-terminates with a foot shock $(0.4 \mathrm{~mA}, 1 \mathrm{~s})$. The inter-tone interval was $40 \mathrm{~s}$ and the conditioning session lasted $5 \mathrm{~min} 30 \mathrm{~s}$ in total.

\subsubsection{AFC extinction training}

One day after AFC training, rats were introduced into a box displaying a new context, Context B (green walls of rough texture, gray plastic floor covered with flocks, cleaned with $4 \%$ chlorine), for $25 \mathrm{~min}$. During the test the rats were exposed to conditioned tone three times for 5,5 and 3 min (with 5 min silence between tones) without electrical shocks. 


\subsection{Measurement of brain metabolism (2-deoxyglucose} autoradiography)

In Experiment 1 (AFC), at 47 days of age, rats received an intraperitoneal injection of (14C)-2-deoxyglucose and were returned to their home cage for $18 \mathrm{~min}$ followed by AFC test for $23 \mathrm{~min}$ right before being sacrificed. In Experiment 2 (basal), at 47 days of age, rats received an intraperitoneal injection of (14C)-2-deoxyglucose and were returned to their home cage and sacrificed $45 \mathrm{~min}$ after injection. In all cases the brains were rapidly extracted $45 \mathrm{~min}$ after 2DG injection and stored at $-80 \mathrm{C}$ until sectioning. Brains were sliced in $20 \mu \mathrm{m}$ coronal sections, collected every $60 \mu \mathrm{m}$ and then processed for autoradiography together with ${ }^{14} \mathrm{C}$ Micro-scale autoradiography standards (RPA504 GE Healthcare). After exposure films were developed and the brain sections stained with cresyl violet. Images of the brain slices were acquired and registered to their corresponding autoradiograms using the MCID acquisition system. Using the MCID software, the average optical density of the sub-regions of the hippocampus prefrontal cortex, hypothalamus and amygdaloid complex were determined and their corresponding radioactive signal determined. Regions were based on Paxinos \& Watson (1998). After we verified that there were no between- group differences in whole-brain 2DG uptake, regional metabolic rate was normalized using whole-brain 2DG values to compensate for variations in 2DG incorporation across individuals.

\subsection{Statistics}

Data was analyzed using two-way ANOVA. When the sex $*$ treatment interaction was significant, Student's $t$-tests were performed as post hoc analysis. Pearson's correlation was employed to study the relationship between metabolic rate in the different brain regions or the association between the regional metabolic rate and behavior during AFC test. PASWStatistics 18.0 statistical package was used for the analyses. Data represent the mean \pm S.E.M. For all tests, null hypotheses were rejected at a probability level of $p<0.05$. Due to the large number of groups and treatments the complexity of our data is substantial. Consequently, we chose to focus here on the effects of peri-pubertal stress in behavior and brain metabolism in rats undergoing fear-conditioning extinction, with a separate analysis for rats that did not undergo fear conditioning.

\section{Results}

3.1. Peri-pubertal stress influences behavior during fear conditioning acquisition and extinction training

Four days after the end of the application of the stress protocol (i.e., 46 days of age), rats were conditioned to fear by pairing the end of a previously neutral tone with a mild foot-shock. Twoway ANOVA revealed a significant treatment effect in the behavior of the rats before, during and after the tone-shock pairings. Animals stressed during adolescence took longer time to freeze $[F(1$, 31 ) $=12.17 p=0.001]$ (Fig. 1A) and spent less time freezing before the shocks $[F(1,31)=4.74 p=0.037]$ (Fig. 1B), during the tones $[F$ $(1,31)=8.16 p=0.008$ ] (Fig. 1C) and after the tone-shock pairings $[F(1,31)=5.88 p=0.021]$ (Fig. 1D). No sex differences or sex $*$ treatment interactions were found in these tests.

Twenty-four hours after training, we studied the responses to an initial fear extinction training by placing the rats in a novel environment and re-exposing them to the trained tone, without additional foot-shocks. Two-way ANOVA revealed that peri-pubertal stress interacted with sex vis-à-vis the time the rats spent freezing during both the entire extinction test $[F(1,31)=7.57 p=0.010]$
(Fig. 1E) and during the tones $[F(1,31)=8.26 p=0.007]$ (Fig. 1G) Post hoc comparisons indicated that: a) stressed females showed a significant lower freezing during extinction than stressed males [ $t$ $(16)=2.158 p=0.046]$ (Fig. 1E) and control females $[t(15)=3.319$ $p=0.005$ ] (Fig. 1E); and b) stressed males froze less than control males during the tones $[t(16)=-2.379 p=0.030]$ (Fig. $1 G$ ). Animals in the two stress groups showed bigger latencies to freeze than those in the control groups, regardless of sex $[F(1$, $29)=7.24 p=0.012$ ] (Fig. 1F). No sex differences were found in these tests. Finally there were no significant differences in the freezing of the animals between tones.

\subsection{Animals stressed during puberty showed higher metabolic rate in different brain regions during extinction training}

Next, we studied whether the behavioral differences during extinction could be associated to differential metabolic activation of specific brain regions. For this purpose, animals were injected with 2DG before extinction training and their brains were processed right after training. We studied the metabolic rate in brain regions previously shown to be involved in emotional control and memory, including frontal cortex (cingulate, insular, orbital, prelimbic, infralimbic and motor cortices); hippocampus (entire hippocampus, CA1, CA3 and dentate gyrus); amygdala (basolateral, basal, lateral and central amygdala) and the paraventricular nucleus of the hypothalamus (Supplementary Fig. 1). Two-way ANOVA revealed that peri-pubertal stress was associated with a higher metabolic rate in multiple brain regions of animals undergoing extinction. Interestingly, it seems that this effect was stronger in females, which (except for the central amygdala) showed a tendency to higher metabolic rate if exposed to stress (see Figs. 2-4).

\subsubsection{Prefrontal cortex}

There was a significant effect of stress (higher 2DG uptake in stressed animals) in: (a) cingulate cortex $[F(1,30)=6.52 p=$ $0.016]$ (Fig. 2A) and (b) motor cortex $[F(1,31)=5.34 p=0.028]$ (Fig. 2F). A two-way ANOVA also revealed a significant sex effect. Stressed (vs. non-stressed) female rats displayed higher 2DG uptake in: (a) cingulate cortex $[F(1,30)=9.06 p=0.005]$ (Fig. $2 \mathrm{~A}$ ); (b) infralimbic cortex $[F(1,30)=4.63 p=0.040]$ (Fig. 2B) and prelimbic cortex $[F(1,30)=4.65 p=0.039]$ (Fig. 2C). No treatment $*$ sex interactions were found.

\subsubsection{Hippocampus}

There was a significant treatment effect (higher 2DG uptake in stressed animals) in: (a) whole hippocampus $[F(1,31)=9.56$ $p=0.004]$ (Fig. 3A); (b) CA1 [F (1, 31) = $7.93 p=0.008]$ (Fig. 3B); (c) CA3 $[F(1,31)=4.59 p=0.040]$ (Fig. 3C) and (d) Dentate Gyrus $[F$ $(1,31)=10.34 p=0.003$ ] (Fig. 3D). No sex differences were observed although a significant treatment $*$ sex interaction was observed in the Dentate Gyrus $[F(1,31)=4.91, p=0.034]$. Post hoc comparisons indicated that within the females, stressed (vs. non-stressed) rats showed a higher 2DG uptake $[t(15)=-3.471 p=0.003]$ (Fig. 3D).

\subsubsection{Amygdala}

There was a significant effect of stress in the metabolic rate in basal amygdala with higher 2DG uptake in stressed animals $[F(1$, 31 ) $=4.32 p=0.046$ ] (Fig. 4B). No sex differences or treatment $*$ sex interaction were observed.

\subsubsection{Paraventricular nucleus (PVNs)}

No sex differences or treatment $*$ sex interaction were observed in the 2DG uptake at the PVN. There was a trend towards lower metabolic rate in the PVN of stressed animals $[F(1,32)=3.37 p=0.082$ control males $0.642 \pm 0.006$; stressed males $0.628 \pm 0.020$; control females $0.609 \pm 0.012$; stressed females $0.578 \pm 0.038$ ] 
A

Training - Latency to freeze

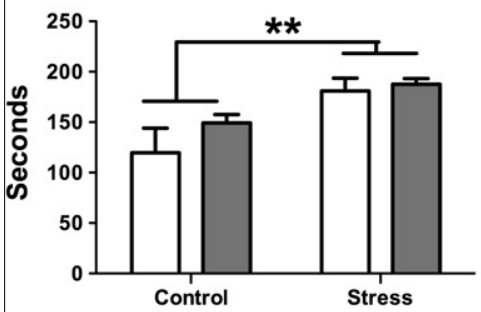

C

Training - Freezing during tones

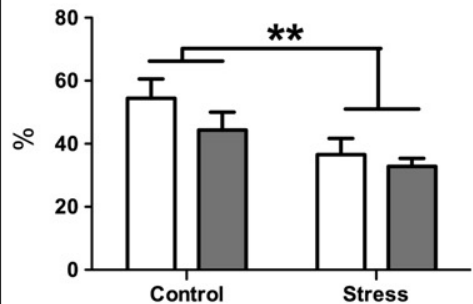

E

Extinction - Freezing (entire test)

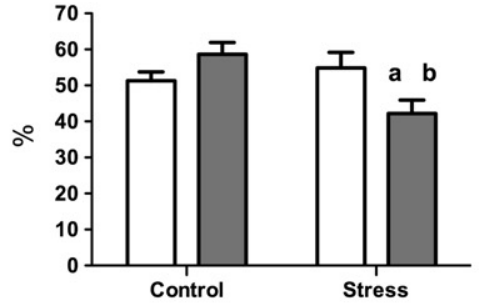

G Extinction - Freezing

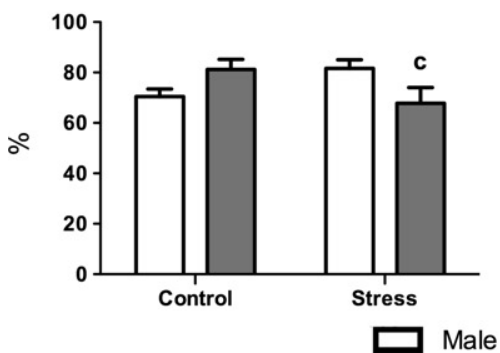

B Training - Freezing before shocks

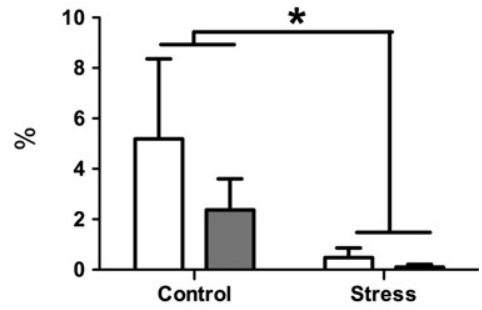

D Training - Freezing after tones

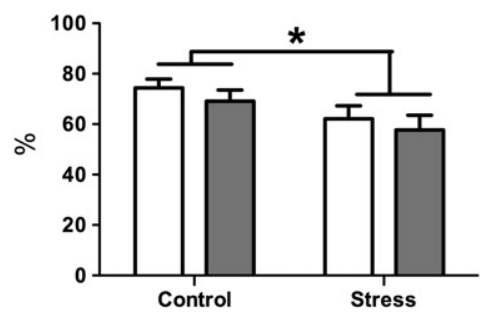

F Extinction - Latency to freeze

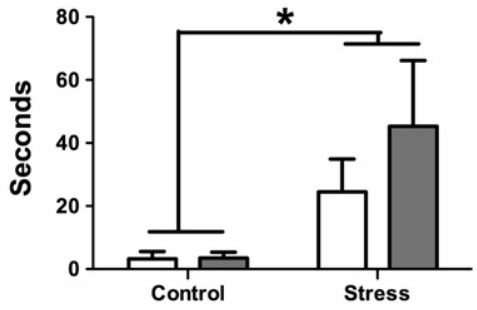

H Extinction - Freezing between tones

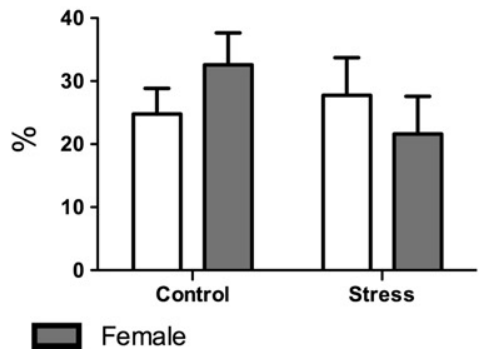

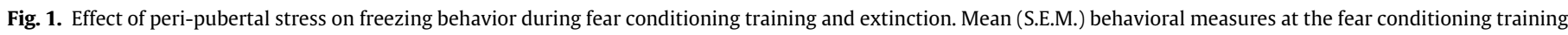

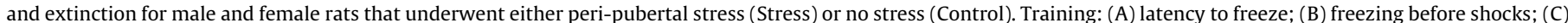

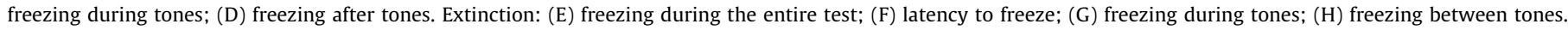

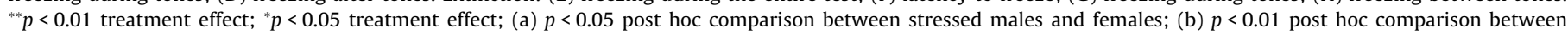
stressed and control females; (c) $p<0.05$ post hoc comparison between stressed and control males.

\subsection{Correlational analyses between metabolic rate in specific brain regions and behavior during extinction training}

Next, we analyzed whether there was any direct relation between behavior and metabolic rate in different brain regions during extinction training. For this purpose we correlated the time spent freezing during tones with the metabolic rate in the different brain regions for each of the experimental groups. The results showed that only stressed males showed a significant positive correlation between percentage of time freezing during the tone and the metabolic rate in the hippocampus, basolateral amygdala and PVN (Table 1). We also observed that, during extinction, the metabolic rate in the hippocampus correlated positively with that in the basolateral amygdala in stressed males and females $(R=0.86$ $p<0.000 ; R=0.70 p<0.009$ respectively; data not shown). Freezing during the tone in stressed males was not correlated with the metabolic rate of any of the prefrontal cortical areas studied (Table 1). Interestingly, there was a significant negative correlation between the time freezing during tones and the metabolic rate of the infralimbic cortex in the control males (Table 1). Neither 

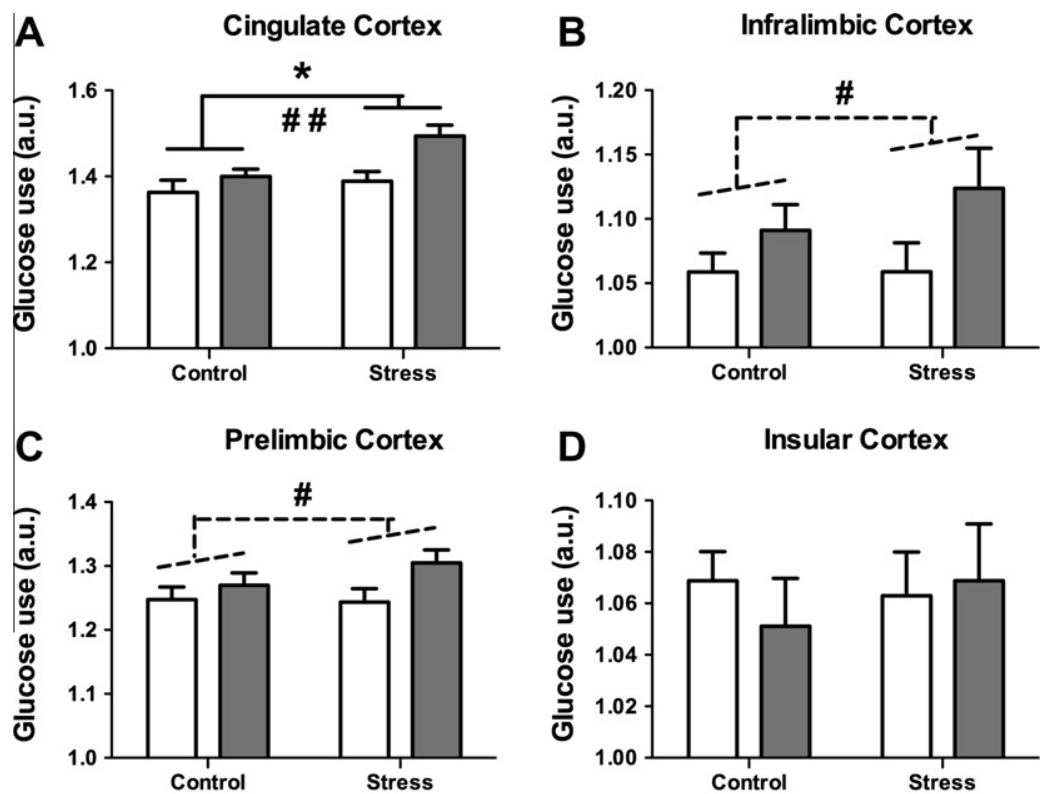

D Insular Cortex
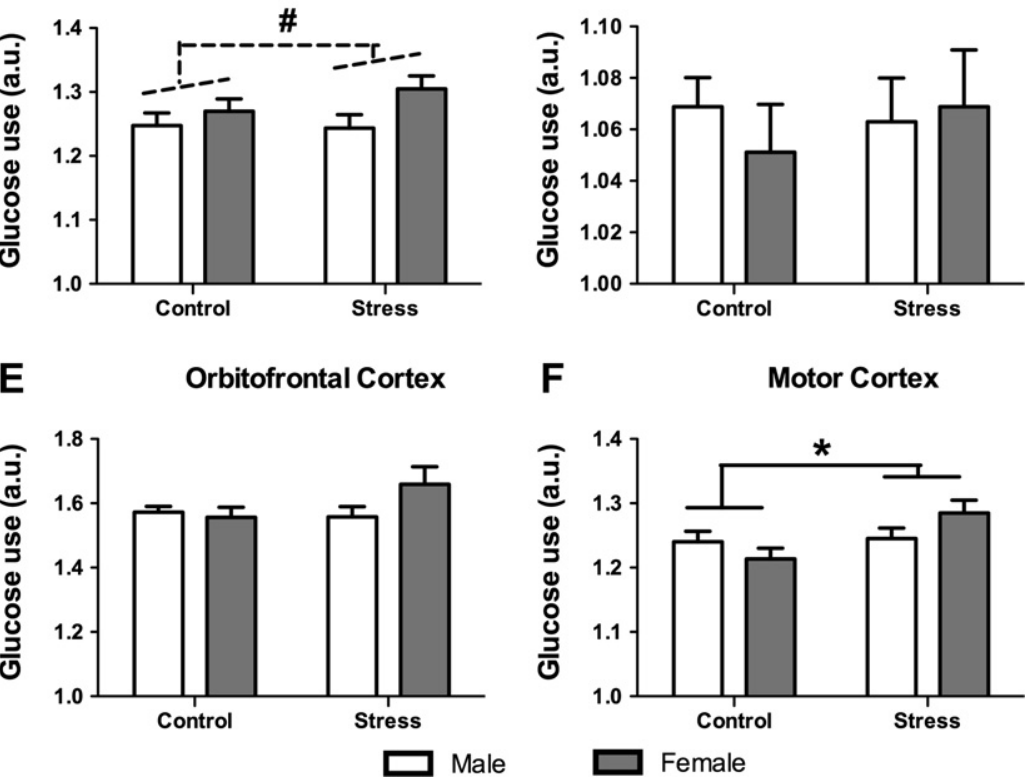

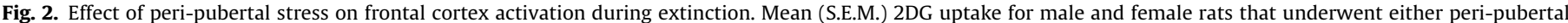

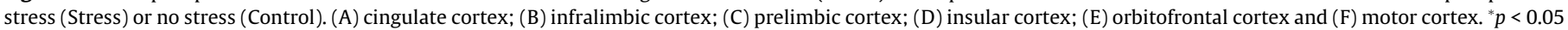
treatment effect; ${ }^{\#} p<0.05$ sex effect; ${ }^{\# \#} p<0.01$ sex effect.
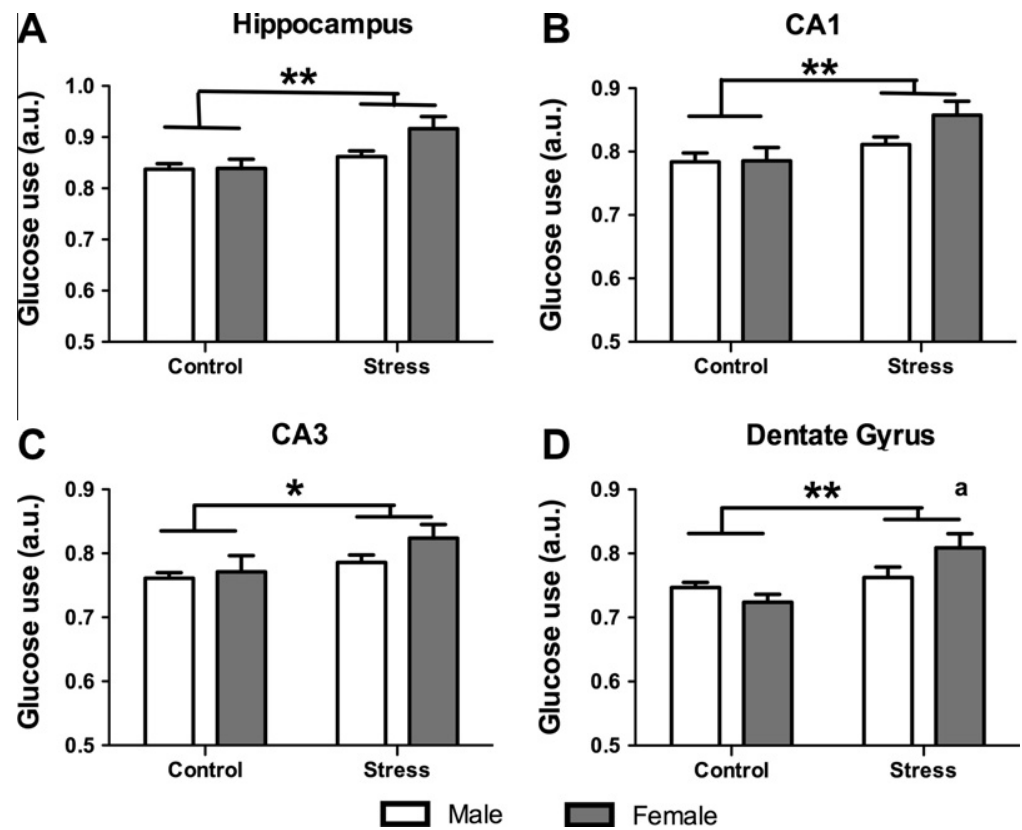

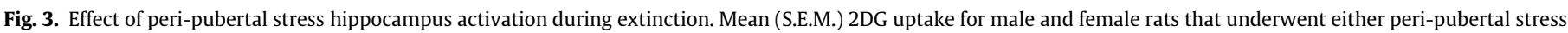

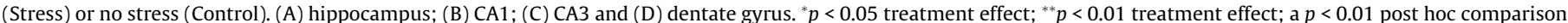
between stressed and control females. 

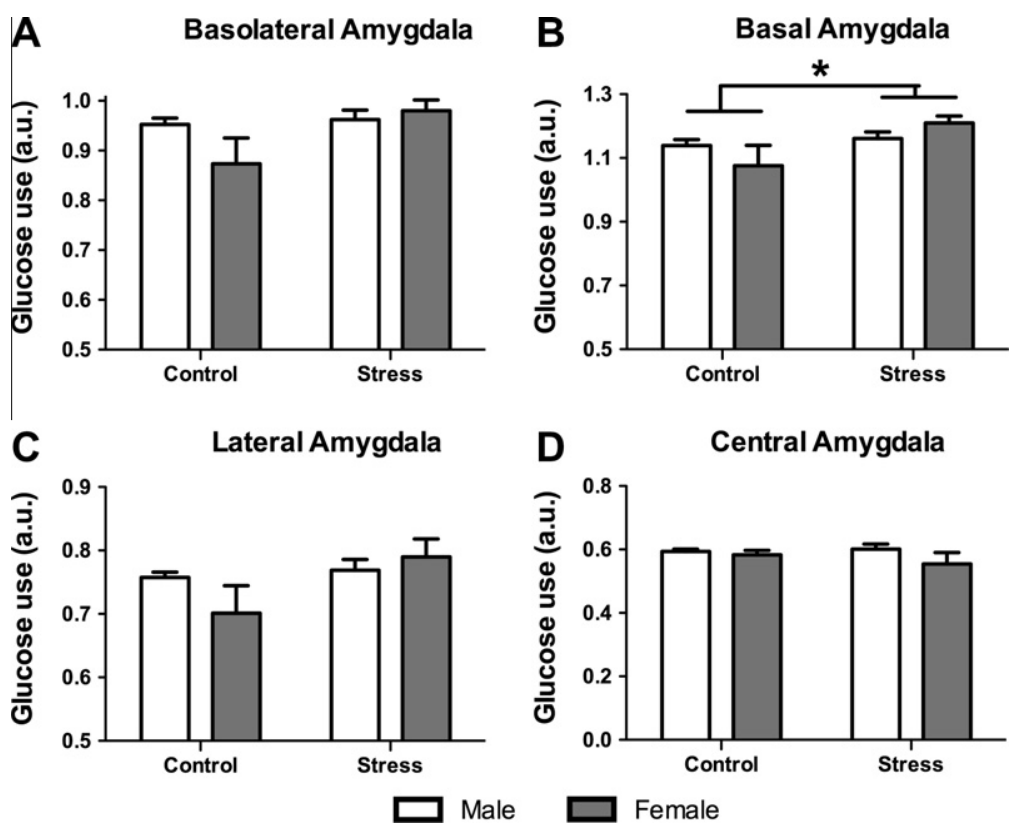

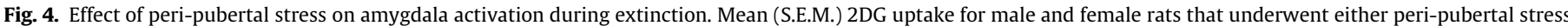
(Stress) or no stress (Control). (A) basolateral amygdala; (B) basal amygdala; (C) lateral amygdala; (D) central amygdala. ${ }^{*} p<0.05$ treatment effect.

Table 1

Pearson correlation between the individual time spent freezing during tones and the activation of different brain areas.

\begin{tabular}{|c|c|c|c|c|c|c|c|c|c|c|c|c|c|c|c|}
\hline & Cg1 & PreLim & IL & Orb & Insular & Motor & $\mathrm{Hp}$ & CA1 & CA3 & DG & BLA & BA & LA & $\mathrm{Ce}$ & PVN \\
\hline \multicolumn{16}{|l|}{ Control females } \\
\hline Pearson correlation & 0.003 & 0.244 & 0.155 & -0.287 & -0.017 & -0.355 & -0.126 & -0.192 & 0.106 & 0.010 & -0.041 & -0.081 & 0.063 & 0.105 & 0.155 \\
\hline $\mathrm{R} 2$ & 0.000 & 0.059 & 0.024 & 0.082 & 0.000 & 0.126 & 0.016 & 0.037 & 0.011 & 0.000 & 0.002 & 0.007 & 0.004 & 0.011 & 0.024 \\
\hline Sig. (2-tailed) & 0.995 & 0.527 & 0.691 & 0.454 & 0.965 & 0.349 & 0.746 & 0.621 & 0.787 & 0.979 & 0.916 & 0.836 & 0.871 & 0.788 & 0.690 \\
\hline $\mathrm{N}$ & 9 & 9 & 9 & 9 & 9 & 9 & 9 & 9 & 9 & 9 & 9 & 9 & 9 & 9 & 9 \\
\hline \multicolumn{16}{|l|}{ Control males } \\
\hline Pearson correlation & -0.238 & -0.651 & -0.801 & -0.546 & 0.061 & -0.630 & -0.221 & -0.231 & 0.135 & -0.340 & 0.472 & 0.407 & 0.733 & 0.300 & 0.304 \\
\hline $\mathrm{R} 2$ & 0.057 & 0.424 & 0.642 & 0.298 & 0.004 & 0.397 & 0.049 & 0.053 & 0.018 & 0.116 & 0.223 & 0.166 & 0.537 & 0.090 & 0.092 \\
\hline Sig. (2-tailed) & 0.570 & 0.080 & $0.017^{*}$ & 0.161 & 0.886 & 0.094 & 0.599 & 0.583 & 0.749 & 0.410 & 0.238 & 0.317 & $0.039^{*}$ & 0.471 & 0.464 \\
\hline $\mathrm{N}$ & 8 & 8 & 8 & 8 & 8 & 8 & 8 & 8 & 8 & 8 & 8 & 8 & 8 & 8 & 8 \\
\hline \multicolumn{16}{|l|}{ Stress females } \\
\hline Pearson correlation & -0.063 & 0.033 & 0.084 & 0.389 & -0.066 & -0.180 & 0.062 & 0.125 & -0.140 & 0.145 & 0.359 & 0.243 & 0.554 & -0.511 & -0.167 \\
\hline $\mathrm{R} 2$ & 0.004 & 0.001 & 0.007 & 0.152 & 0.004 & 0.032 & 0.004 & 0.016 & 0.020 & 0.021 & 0.129 & 0.059 & 0.307 & 0.261 & 0.028 \\
\hline Sig. (2-tailed) & 0.882 & 0.939 & 0.842 & 0.340 & 0.876 & 0.669 & 0.885 & 0.769 & 0.741 & 0.732 & 0.382 & 0.562 & 0.154 & 0.196 & 0.693 \\
\hline $\mathrm{N}$ & 8 & 8 & 8 & 8 & 8 & 8 & 8 & 8 & 8 & 8 & 8 & 8 & 8 & 8 & 8 \\
\hline \multicolumn{16}{|l|}{ Stress males } \\
\hline Pearson correlation & -0.214 & 0.037 & 0.217 & -0.628 & -0.096 & -0.598 & 0.925 & 0.805 & 0.820 & 0.759 & 0.867 & 0.661 & 0.765 & 0.759 & 0.846 \\
\hline $\mathrm{R} 2$ & 0.046 & 0.001 & 0.047 & 0.395 & 0.009 & 0.358 & 0.856 & 0.648 & 0.672 & 0.576 & 0.752 & 0.437 & 0.585 & 0.576 & 0.716 \\
\hline Sig. (2-tailed) & 0.581 & 0.924 & 0.575 & 0.052 & 0.793 & 0.068 & $0.000^{* * *}$ & $0.005^{* *}$ & $0.005^{* *}$ & $0.011^{* *}$ & $0.001^{* *}$ & $0.037^{*}$ & $0.01^{* *}$ & $0.011^{*}$ & $0.002^{* *}$ \\
\hline $\mathrm{N}$ & 9 & 9 & 9 & 10 & 10 & 10 & 10 & 10 & 10 & 10 & 10 & 10 & 10 & 10 & 10 \\
\hline
\end{tabular}

${ }^{*} p<0.05$.

*** $p<0.01$.

*** $p<0.001$.

stressed nor control females showed any correlation between the metabolic rate in any of the brain regions and the percentage of freezing during tones (Table 1 ).

\subsection{Peri-pubertal stress does not affect brain metabolic rate under} basal conditions

Finally, we determined whether the observed differences in brain metabolism between the stressed and non-stressed animals were specific to the state of extinction or present also when the rats stayed undisturbed in their home cages. For this purpose,
5 days after the end of the stress period (i.e., 47 days of age), stressed and control animals were injected with radioactive 2DG and returned to their home cages until decapitation 45 min later. We studied the same brain regions as it was the case for the extinction experiments (i.e. prefrontal cortex, hippocampus, amygdala and PVN). Two-way ANOVA analysis revealed no significant stress effect or stress $*$ sex interaction in any of the brain regions (Table 2). There was a significant sex effect in the metabolic rate in the entire hippocampus and its sub-regions (CA1, CA3 and dentate gyrus) with females displaying higher metabolic rate than males (Table 2). 
Table 2

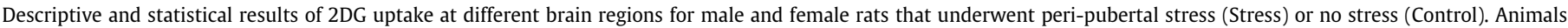
were resting at their home cage.

\begin{tabular}{|c|c|c|c|c|c|c|c|c|c|c|}
\hline \multirow[t]{3}{*}{ Brain area } & \multirow[t]{3}{*}{ Abbreviation } & \multirow[t]{3}{*}{ Bergma* } & \multicolumn{4}{|l|}{ Groups } & \multicolumn{4}{|c|}{ Statistical analysis } \\
\hline & & & \multicolumn{2}{|l|}{ Control } & \multicolumn{2}{|l|}{ Stressed } & \multirow{2}{*}{$\begin{array}{l}\text { Factor } \\
\text { treat } \\
\text { F }\end{array}$} & \multirow{2}{*}{$\begin{array}{l}\text { Factor } \\
\text { sex } \\
\text { F }\end{array}$} & \multirow{2}{*}{$\begin{array}{l}\text { Interaction } \\
(\text { sex } * \text { treat }) \\
\mathrm{F}\end{array}$} & \multirow{2}{*}{$\begin{array}{l}\text { Degrees of } \\
\text { freedom }\end{array}$} \\
\hline & & & $\begin{array}{l}\text { Females } \\
(n=8-9)\end{array}$ & $\begin{array}{l}\text { Males } \\
(n=8-10)\end{array}$ & $\begin{array}{l}\text { Females } \\
(n=9)\end{array}$ & $\begin{array}{l}\text { Males } \\
(n=8)\end{array}$ & & & & \\
\hline Cingulate cortex & $\mathrm{Cg}$ & 3.20 & $1.42(0.10)$ & $1.40(0.10)$ & $1.55(0.10)$ & $1.35(0.08)$ & 0.187 & 1.227 & 0.777 & $(1,31)$ \\
\hline Prelimbic cortex & PrL & 3.20 & $1.33(0.07)$ & $1.27(0.09)$ & $1.37(0.09)$ & $1.21(0.08)$ & 0.021 & 1.809 & 0.459 & $(1,31)$ \\
\hline Infralimbic cortex & IL & 3.20 & $1.17(0.07)$ & $1.13(0.07)$ & $1.20(0.07)$ & $1.04(0.06)$ & 0.179 & 2.110 & 0.865 & $(1,31)$ \\
\hline Orbitofrontal cortex & Orb & 3.20 & $1.70(0.13)$ & $1.61(0.12)$ & $1.80(0.14)$ & $1.58(0.12)$ & 0.078 & 1.501 & 0.319 & $(1,31)$ \\
\hline Insular cortex & Ins & 3.20 & $1.25(0.06)$ & $1.19(0.07)$ & $1.24(0.08)$ & $1.15(0.06)$ & 0.138 & 1.231 & 0.094 & $(1,31)$ \\
\hline Motor cortex & MC & 3.20 & $1.38(0.09)$ & $1.36(0.09)$ & $1.52(0.09)$ & $1.38(0.08)$ & 0.816 & 0.788 & 0.384 & $(1,30)$ \\
\hline Hippocampus & $\mathrm{HC}$ & -3.60 & $1.01(0.06)$ & $0.95(0.04)$ & $1.06(0.06)$ & $0.89(0.07)$ & 0.014 & $4.670^{*}$ & 0.957 & $(1,31)$ \\
\hline CA1 & CA1 & -3.60 & $0.95(0.05)$ & $0.90(0.03)$ & $1.00(0.05)$ & $0.84(0.05)$ & 0.011 & $4.520^{*}$ & 1.329 & $(1,31)$ \\
\hline CA3 & CA3 & -3.60 & $0.97(0.06)$ & $0.90(0.06)$ & $0.99(0.05)$ & $0.82(0.06)$ & 0.223 & $4.399^{*}$ & 0.660 & $(1,31)$ \\
\hline Dentate gyrus & DG & -3.60 & $1.83(0.09)$ & $1.70(0.08)$ & $1.91(0.12)$ & $1.57(0.11)$ & 0.107 & $5.610^{*}$ & 1.112 & \\
\hline Balolateral amygdala & BLA & -3.30 & $1.04(0.07)$ & $0.97(0.06)$ & $1.13(0.08)$ & $1.01(0.08)$ & 0.732 & 1.668 & 0.140 & $(1,30)$ \\
\hline Basal amygdala & BA & -3.30 & $1.24(0.10)$ & $1.15(0.08)$ & $1.39(0.11)$ & $1.22(0.11)$ & 1.195 & 1.822 & 0.137 & $(1,30)$ \\
\hline Lateral amigdala & LA & -3.30 & $0.87(0.04)$ & $0.81(0.04)$ & $0.90(0.06)$ & $0.79(0.06)$ & 0.003 & 2.750 & 0.343 & $(1,30)$ \\
\hline Central amygdala & $\mathrm{CeA}$ & -2.12 & $0.72(0.03)$ & $0.67(0.03)$ & $0.71(0.06)$ & $0.61(0.04)$ & 0.610 & 3.437 & 0.336 & $(1,31)$ \\
\hline PVN & PVN & -1.40 & $0.72(0.04)$ & $0.67(0.04)$ & $0.69(0.05)$ & $0.63(0.05)$ & 0.570 & 1.499 & 0.038 & $(1,31)$ \\
\hline
\end{tabular}

Sex differences in brain 2DG uptake (mean \pm S.E.M.) among stressed and control rats resting at their homecage.

$p<0.05$ sex effect.

\section{Discussion}

Using glucose metabolism as an indicator of brain activity, we have studied the differences in regional brain activity associated with the exposure to stress right before and during puberty in two situations: (a) during extinction of auditory fear conditioning or (b) while the animals rest in their home cages. We have also studied the correlations between the metabolic activation in different brain structures and the animals' behavior during extinction training.

\subsection{Peri-pubertal stress impacts brain metabolism during fear extinction training}

Our initial hypothesis was that peri-pubertal stress would cause changes in regional brain metabolism during the processing of learned fear stimuli, namely during extinction. We based this hypothesis on recent work showing that peri-pubertal stress reduces anxiety in the rat (Toledo-Rodriguez \& Sandi, 2011). In agreement with our hypothesis, ANOVA of 2DG uptake data during extinction revealed a higher metabolic activation in the cingulate and motor cortices, hippocampus, CA1, CA3 and basal amygdala of animals that underwent peri-pubertal stress when fear learning and extinction were given shortly afterwards, during adolescence. This difference between the stressed and non-stressed rats was specific for extinction, as it was not observed in the rats that stayed undisturbed in their home cage. These results are in line with the known anatomical architecture of extinction and the structural changes these regions undergo when adult animals are exposed to stress (Holmes \& Wellman, 2009; Vyas, Mitra, Shankaranarayana Rao, \& Chattarji, 2002; Watanabe, Gould, \& McEwen, 1992). Fear extinction is an active learning process mediated by a large-scale brain network including the prefrontal cortex, hippocampus and amygdala (Herry et al., 2010). Recent studies indicate that close interactions between these brain regions contribute to distinct extinction processes, with learning mediated by cellular networks centered on the basal amygdala and consolidation recruiting subregions of the PFC and hippocampus (Quirk \& Mueller, 2008). For example, inactivation of the basal amygdala completely blocks acquisition of extinction (Herry et al., 2008) and c-fos induction in the basal amygdala is strongly reduced in animal models of impaired extinction learning (Muigg et al., 2008). Likewise higher amygdala activation during extinction learning has been described in a recent human imaging study investigating PTSD patients (Milad et al., 2009). In our case, stress lead to a significant raise of basal amygdala activation independently of the behavior during extinction (i.e. stressed males froze more than control males and stressed females froze less than their controls during extinction). Thus it seems that peri-pubertal stress uncouples the correlation between the metabolic activation of the basal amygdala and extinction acquisition seen in non-stressed subjects. Additionally, we also showed that animals exposed to stress during adolescence displayed higher hippocampal activation regardless of their behavior during extinction. This finding may seem surprising since hippocampal function is clearly essential for contextual fear learning and we were studying cue learning. Yet, extinction of cue memories is context dependent and hippocampal activation is crucial for consolidation (transfer from short term to long term memory) of the extinguished memories (Bruchey et al., 2007). Therefore it seems that, in addition to uncoupling the correlation between basal amygdala activation and extinction acquisition, stress during adolescence may also lead to hyper-activation of the hippocampus when the animal undergoes learning in new context. Further studies should investigate these phenomena.

\subsection{Sex-related differences in brain metabolic activation during extinction training}

The majority of previous rodent studies of metabolic activation during extinction focused on males (Bruchey et al., 2007; Hefner et al., 2008; Herry \& Mons, 2004; Muigg et al., 2008). The use of male and female rats in our study enabled us to investigate whether the effects of peri-pubertal stress were sex-specific. Our study replicates the lower activation of the infralimbic cortex in non-stressed males with impaired AFC extinction (Barrett, Shumake, Jones, \& Gonzalez-Lima, 2003; Hefner et al., 2008; Herry \& Garcia, 2002; Muigg et al., 2008). Yet we expand that knowledge by showing that the correlation between infralimbic cortex activation \& extinction is not found in control females or in animals of both sexes previously exposed to stress. This lack of correlation could be explained by previous research (performed in adult males) showing that exposure to stress reduces the dendritic arbor and spine number of pyramidal neurons in the medial prefrontal cortex (Radley et al., 2006). Moreover the dendritic reduction in 
the infralimbic cortex is accompanied with deficits in extinction of cue fear conditioning (Izquierdo, Wellman, \& Holmes, 2006). To our knowledge, no study has investigated the effects of stress on infralimbic neuronal architecture in female rats. Nevertheless, a recent study showed higher morphological complexity of pyramidal neurons in the anterior cingulate and prelimbic cortices when adult female rats are exposed to chronic stress (Garrett \& Wellman, 2009). Yet, we should bear in mind that the current knowledge of the structural changes in the prefrontal cortex induced by exposure to stress came from studies in adult animals.

Additionally, correlational analysis of our data revealed that only stressed males showed a significant association between freezing during tone and the metabolic activation of hippocampus, basolateral amygdala and PVN. These results are consistent with previous studies showing a synchronization of the theta frequency range between basolateral amygdala and hippocampus during fear memory retrieval (Seidenbecher, Laxmi, Stork, \& Pape, 2003) that declines during extinction learning (Lesting et al., 2011). Indeed, in our case we found a highly significant correlation between the metabolic activation of the hippocampus and the basolateral amygdala during extinction in stressed males and females, but not in the non-stressed groups.

Paradoxically, during extinction stressed female rats showed higher metabolic activation in the limbic regions and lower freezing levels than control females, while stressed males froze for longer time but showed barely any difference in the regional brain metabolism. Indeed the higher metabolic activation in stressed (vs. non-stressed) animals during extinction was mainly driven by females; in most regions, 2DG uptake was not different between the stressed and non-stressed males. This phenomenon was more evident in the dentate gyrus. Additionally, we found that, under basal conditions (i.e. animals resting at their home cage) females showed higher metabolic activation in the hippocampus. The difference was magnified by previous exposure to peri-pubertal stress. These results are consistent with previous studies comparing 2DG uptake or c-fos expression in adult male vs. female rats exposed to acute physical stress (Brown, Siegel, \& Etgen, 1996; Ceccarelli, Scaramuzzino, \& Aloisi, 1999).

Finally, we also showed that stressed females displayed a higher metabolic activation of the motor cortex than their controls or both groups of males. This might simply reflect the higher locomotor activity (i.e., less freezing) of stressed females during extinction.

\subsection{Peri-pubertal stress has a sex-dependent effect on extinction training}

Another significant finding from our study is the differential effects of sex and stress on the behavior during acquisition and extinction of fear conditioning. While peri-pubertal stress reduced freezing during fear conditioning training in both males and females, it had a sex-specific effect on the behavior of the animals during extinction. Similar to our results, exposure to chronic stress during adulthood has been shown to impair extinction to the tone in males, but facilitate extinction in females (Baran, Armstrong, Niren, Hanna, \& Conrad, 2009). Likewise, male rats exposed to juvenile stress (P25-29) froze more than controls during adult fear conditioning (Tsoory, Guterman, \& Richter-Levin, 2010). Yet, a recent study showed that male mice exposed to juvenile stress froze less during fear conditioning training and extinction when the animals were tested before puberty (Peleg-Raibstein \& Feldon, 2011) but not when fear conditioning was performed in adulthood. Other early life manipulations (neonatal maternal separation and handling) often lead to impairment in fear conditioning in adulthood (Kosten, Lee, \& Kim, 2006; Lehmann, Pryce, Bettschen, \& Feldon, 1999; Meerlo, Horvath, Nagy, Bohus, \& Koolhaas, 1999; Pryce,
Bettschen, Nanz-Bahr, \& Feldon, 2003). Interestingly, these sex differences on the impact of stress also expand to other forms of learning, although the effects are reversed for cued and spatial learning. Similar to our results, acute stress exposure impairs classical eye-blink conditioning in female rats while enhancing performance in males (Wood \& Shors, 1998). On the other hand, stress impairs performance in the $\mathrm{Y}$ maze and radial arm maze in males but not in females (Bowman, Beck, \& Luine, 2003; Conrad, Grote, Hobbs, \& Ferayorni, 2003).

\section{Conclusion}

In conclusion, here we show that while peri-pubertal stress reduces freezing during fear conditioning training in both sexes, it had a sex-specific effect during extinction. Moreover the metabolic activation during extinction in hippocampus, basal amygdala and cingulate and motor cortices was higher in animals exposed to peri-pubertal stress. This stress-related difference was specific for extinction, as it did not happen when the animals stayed undisturbed in their home cage. Interestingly, metabolic activation of the infralimbic cortex was negatively correlated with freezing during extinction only in control males, while only males stressed during puberty showed a significant correlation between behavior during extinction and metabolic activation of hippocampus, amygdala and paraventricular nucleus. No correlations between regional brain metabolism and behavior during extinction were observed in females (control or stress). These results suggest that exposure to stress during puberty modulates, in a sex-specific way, metabolic brain activation, learning and behavior in response to subsequent challenges. Moreover, these results emphasize that data obtained in males cannot always be extrapolated to females, particularly on the resilience and/or vulnerability to stress.

\section{Acknowledgments}

We would like to thank Miss Coralie Siegmund and Ms Clara Rosetti for their invaluable experimental assistance, Dr. Cristina Marquez for help with the behavioral analyses and brain harvesting and Dr. Julia Parafita for training on the 2DG metabolic mapping technique and MCID analysis. We also would like to thank Prof. Kevin Fone and Prof. Terence Parker for their useful comments on the manuscript. This work was supported by the Roche Research Foundation, and grants from the EU 7th Framework Program (MemStick), the Swiss National Science Foundation 310000-120791; Sinergia CRSIK0-122697; CRSIK0-122691 and the NCCR Synapsy.

\section{Appendix A. Supplementary material}

Supplementary data associated with this article can be found, in the online version, at http://dx.doi.org/10.1016/j.nlm.2012.05.006.

\section{References}

Avital, A., \& Richter-Levin, G. (2005). Exposure to juvenile stress exacerbates the behavioural consequences of exposure to stress in the adult rat. International Journal of Neuropsychopharmacology, 8(2), 163-173.

Baran, S. E., Armstrong, C. E., Niren, D. C., Hanna, J. J., \& Conrad, C. D. (2009). Chronic stress and sex differences on the recall of fear conditioning and extinction. Neurobiology of Learning and Memory, 91(3), 323-332.

Barrett, D., Shumake, J., Jones, D., \& Gonzalez-Lima, F. (2003). Metabolic mapping of mouse brain activity after extinction of a conditioned emotional response Journal of Neuroscience, 23(13), 5740-5749.

Bowman, R. E., Beck, K. D., \& Luine, V. N. (2003). Chronic stress effects on memory: Sex differences in performance and monoaminergic activity. Hormones and Behavior, 43(1), 48-59.

Brown, L. L., Siegel, H., \& Etgen, A. M. (1996). Global sex differences in stressinduced activation of cerebral metabolism revealed by 2-deoxyglucose autoradiography. Hormones and Behavior, 30(4), 611-617. 
Bruchey, A. K., Shumake, J., \& Gonzalez-Lima, F. (2007). Network model of fear extinction and renewal functional pathways. Neuroscience, 145(2), 423-437.

Ceccarelli, I., Scaramuzzino, A., \& Aloisi, A. M. (1999). Effects of formalin pain on hippocampal c-Fos expression in male and female rats. Pharmacology, Biochemistry and Behavior, 64(4), 797-802.

Conrad, C. D., Grote, K. A., Hobbs, R. J., \& Ferayorni, A. (2003). Sex differences in spatial and non-spatial Y-maze performance after chronic stress. Neurobiology of Learning and Memory, 79(1), 32-40.

Dahl, R. E. (2004). Adolescent brain development: A period of vulnerabilities and opportunities. Keynote address. Annals of the New York Academy of Sciences, 1021, 1-22.

Garrett, J. E., \& Wellman, C. L. (2009). Chronic stress effects on dendritic morphology in medial prefrontal cortex: Sex differences and estrogen dependence. Neuroscience, 162(1), 195-207.

Hefner, K., Whittle, N., Juhasz, J., Norcross, M., Karlsson, R. M., Saksida, L. M., et al (2008). Impaired fear extinction learning and cortico-amygdala circuit abnormalities in a common genetic mouse strain. Journal of Neuroscience, 28(32), 8074-8085.

Heim, C., Plotsky, P. M., \& Nemeroff, C. B. (2004). Importance of studying the contributions of early adverse experience to neurobiological findings in depression. Neuropsychopharmacology, 29(4), 641-648.

Herry, C., Ciocchi, S., Senn, V., Demmou, L., Muller, C., \& Luthi, A. (2008). Switching on and off fear by distinct neuronal circuits. Nature, 454(7204), 600-606.

Herry, C., Ferraguti, F., Singewald, N., Letzkus, J. J., Ehrlich, I., \& Luthi, A. (2010). Neuronal circuits of fear extinction. European Journal of Neuroscience, 31(4), 599-612.

Herry, C., \& Garcia, R. (2002). Prefrontal cortex long-term potentiation, but not longterm depression, is associated with the maintenance of extinction of learned fear in mice. Journal of Neuroscience, 22(2), 577-583.

Herry, C., \& Mons, N. (2004). Resistance to extinction is associated with impaired immediate early gene induction in medial prefrontal cortex and amygdala. European Journal of Neuroscience, 20(3), 781-790.

Holmes, A., \& Wellman, C. L. (2009). Stress-induced prefrontal reorganization and executive dysfunction in rodents. Neuroscience and Biobehavioral Reviews, 33(6), $773-783$.

Isgor, C., Kabbaj, M., Akil, H., \& Watson, S. J. (2004). Delayed effects of chronic variable stress during peripubertal-juvenile period on hippocampal morphology and on cognitive and stress axis functions in rats. Hippocampus, 14(5), 636-648.

Ito, H., Nagano, M., Suzuki, H., \& Murakoshi, T. (2010). Chronic stress enhances synaptic plasticity due to disinhibition in the anterior cingulate cortex and induces hyper-locomotion in mice. Neuropharmacology, 58(4-5), 746-757.

Izquierdo, A., Wellman, C. L., \& Holmes, A. (2006). Brief uncontrollable stress causes dendritic retraction in infralimbic cortex and resistance to fear extinction in mice. Journal of Neuroscience, 26(21), 5733-5738.

Jacobson-Pick, S., \& Richter-Levin, G. (2010). Differential impact of juvenile stress and corticosterone in juvenility and in adulthood, in male and female rats. Behavioural Brain Research, 214(2), 268-276.

Kessler, R. C. (2003). Epidemiology of women and depression. Journal of Affective Disorders, 74(1), 5-13.

Kessler, R. C., \& Magee, W. J. (1993). Childhood adversities and adult depression: basic patterns of association in a US national survey. Psychological Medicine, 23(3), 679-690.

Kosten, T. A., Lee, H. J., \& Kim, J. J. (2006). Early life stress impairs fear conditioning in adult male and female rats. Brain Research, 1087(1), 142-150.

Lehmann, J., Pryce, C. R., Bettschen, D., \& Feldon, J. (1999). The maternal separation paradigm and adult emotionality and cognition in male and female Wistar rats. Pharmacology, Biochemistry and Behavior, 64(4), 705-715.

Lesting, J., Narayanan, R. T., Kluge, C., Sangha, S., Seidenbecher, T., \& Pape, H. C. (2011). Patterns of coupled theta activity in amygdala-hippocampal-prefrontal cortical circuits during fear extinction. PLOS ONE, 6(6), e21714.

Maeng, L. Y., Waddell, J., \& Shors, T. J. (2010). The prefrontal cortex communicates with the amygdala to impair learning after acute stress in females but not in males. Journal of Neuroscience, 30(48), 16188-16196.

Maren, S. (2001). Neurobiology of Pavlovian fear conditioning. Annual Review of Neuroscience, 24, 897-931.

McCormick, C. M., Smith, C., \& Mathews, I. Z. (2008). Effects of chronic social stress in adolescence on anxiety and neuroendocrine response to mild stress in male and female rats. Behavioural Brain Research, 187(2), 228-238.

Meerlo, P., Horvath, K. M., Nagy, G. M., Bohus, B., \& Koolhaas, J. M. (1999). The influence of postnatal handling on adult neuroendocrine and behavioural stress reactivity. Journal of Neuroendocrinology, 11(12), 925-933.

Milad, M. R., Pitman, R. K., Ellis, C. B., Gold, A. L., Shin, L. M., Lasko, N. B., et al. (2009). Neurobiological basis of failure to recall extinction memory in posttraumatic stress disorder. Biological Psychiatry, 66(12), 1075-1082.
Muigg, P., Hetzenauer, A., Hauer, G., Hauschild, M., Gaburro, S., Frank, E., et al. (2008). Impaired extinction of learned fear in rats selectively bred for high anxiety-evidence of altered neuronal processing in prefrontal-amygdala pathways. European Journal of Neuroscience, 28(11), 2299-2309.

Paus, T., Keshavan, M., \& Giedd, J. N. (2008). Why do many psychiatric disorders emerge during adolescence? Nature Reviews Neuroscience, 9(12), 947-957.

Paxinos, G., \& Watson, C. (1998). The rat brain in stereotaxic coordinates (4th ed.). San Diego: Academic Press.

Peleg-Raibstein, D., \& Feldon, J. (2011). Differential effects of post-weaning juvenile stress on the behaviour of C57BL/6 mice in adolescence and adulthood. Psychopharmacology (Berl).

Penza, K. M., Heim, C. \& Nemeroff, C. B. (2003). Neurobiological effects of childhood abuse: Implications for the pathophysiology of depression and anxiety. Archives of Womens Mental Health, 6(1), 15-22.

Pryce, C. R., Bettschen, D., Nanz-Bahr, N. I., \& Feldon, J. (2003). Comparison of the effects of early handling and early deprivation on conditioned stimulus, context, and spatial learning and memory in adult rats. Behavioral Neuroscience, 117(5), 883-893.

Quirk, G. J., \& Mueller, D. (2008). Neural mechanisms of extinction learning and retrieval. Neuropsychopharmacology, 33(1), 56-72.

Radley, J. J., Rocher, A. B., Miller, M., Janssen, W. G., Liston, C., Hof, P. R., et al. (2006). Repeated stress induces dendritic spine loss in the rat medial prefrontal cortex. Cerebral Cortex, 16(3), 313-320.

Rauch, S. L., Shin, L. M., \& Phelps, E. A. (2006). Neurocircuitry models of posttraumatic stress disorder and extinction: human neuroimaging researchpast, present, and future. Biological Psychiatry, 60(4), 376-382.

Sabb, F. W., van Erp, T. G., Hardt, M. E., Dapretto, M., Caplan, R., Cannon, T. D., et al. (2010). Language network dysfunction as a predictor of outcome in youth at clinical high risk for psychosis. Schizophrenia Research, 116(2-3), 173-183.

Seidenbecher, T., Laxmi, T. R., Stork, O., \& Pape, H. C. (2003). Amygdalar and hippocampal theta rhythm synchronization during fear memory retrieval. Science, 301(5634), 846-850.

Sokoloff, L. (1992). Imaging techniques in studies of neural functions. In F. Gonzalez-Lima, T. Finkensadt, \& H. Scheich (Eds.), Advances in metabolic mapping techinques for brain imaging of behavioral and learning functions (pp. 1-37). Boston: Kluwer.

Sokoloff, L., Reivich, M., Kennedy, C., Des Rosiers, M. H., Patlak, C. S., Pettigrew, K. D. et al. (1977). The [14C]deoxyglucose method for the measurement of local cerebral glucose utilization: Theory, procedure, and normal values in the conscious and anesthetized albino rat. Journal of Neurochemistry, 28(5), 897-916.

Spear, L. P. (2000). The adolescent brain and age-related behavioral manifestations. Neuroscience and Biobehavioral Reviews, 24(4), 417-463.

Spear, L. P., \& Brake, S. C. (1983). Periadolescence: Age-dependent behavior and psychopharmacological responsivity in rats. Developmental Psychobiology, 16(2), 83-109.

Steinberg, L., Dahl, R, Keating D., Kupfer, D., Masten, A., \& Pine, D. (2005). The study of developmental psychopathology in adolescence integrating affective neuroscience with the study of context. In D. Cicchetti (Ed.), Handbook of developmental psychopathology. New York: John Wiley \& Sons.

Sterlemann, V., Rammes, G., Wolf, M., Liebl, C., Ganea, K., Muller, M. B., et al. (2010) Chronic social stress during adolescence induces cognitive impairment in aged mice. Hippocampus, 20(4), 540-549.

Styne, D. M. (1994). Physiology of puberty. Adolescent Medicine, 5(1), 171-188.

Tirelli, E., Laviola, G., \& Adriani, W. (2003). Ontogenesis of behavioral sensitization and conditioned place preference induced by psychostimulants in laboratory rodents. Neuroscience and Biobehavioral Reviews, 27(1-2), 163-178.

Toledo-Rodriguez, M., \& Sandi, C. (2011). Stress during adolescence increases novelty seeking and risk-taking behavior in male and female rats. Frontiers in Behavioral Neuroscience, 5, 17.

Tsoory, M. M. Guterman, A. \& Richter-Levin, G. (2010). "Juvenile stress" alters maturation-related changes in expression of the neural cell adhesion molecule L1 in the limbic system: Relevance for stress-related psychopathologies. Journal of Neuroscience Research, 88(2), 369-380.

Vyas, A., Mitra, R., Shankaranarayana Rao, B. S., \& Chattarji, S. (2002). Chronic stress induces contrasting patterns of dendritic remodeling in hippocampal and amygdaloid neurons. Journal of Neuroscience, 22(15), 6810-6818.

Watanabe, Y., Gould, E., \& McEwen, B. S. (1992). Stress induces atrophy of apical dendrites of hippocampal CA3 pyramidal neurons. Brain Research, 588(2), 341-345.

Wood, G. E., \& Shors, T. J. (1998). Stress facilitates classical conditioning in males, but impairs classical conditioning in females through activational effects of ovarian hormones. Proceedings of the National Academy of Sciences of the United States of America - Physical Sciences, 95(7), 4066-4071. 